\title{
Reciprocal Interactions between Epigallocatechin-3-gallate (EGCG) and Human Gut Microbiota In Vitro
}

\author{
Zhibin Liu, Wouter J. C. de Bruijn, Marieke E. Bruins, and Jean-Paul Vincken*
}

Cite This: J. Agric. Food Chem. 2020, 68, 9804-9815

Read Online

ABSTRACT: Interaction of tea phenolics with gut microbiota may play an integral role in the health benefits of these bioactive compounds, yet this interaction is not fully understood. Here, the metabolic fate of epigallocatechin-3-gallate (EGCG) and its impact on gut microbiota were integrally investigated via in vitro fermentation. As revealed by ultrahigh performance liquid chromatography hybrid quadrupole Orbitrap mass spectrometry (UHPLC-Q-Orbitrap-MS), EGCG was promptly degraded into a series of metabolites, including 4-phenylbutyric acid, 3-(3',4'-dihydroxyphenyl)propionic acid, and 3-(4'-hydroxyphenyl)propionic acid, through consecutive ester hydrolysis, C-ring opening, A-ring fission, dehydroxylation, and aliphatic chain shortening. Microbiome profiling indicated that, compared to the blank, EGCG treatment resulted in stimulation of the beneficial bacteria Bacteroides, Christensenellaceae, and Bifidobacterium. Additionally, the pathogenic bacteria Fusobacterium varium, Bilophila, and Enterobacteriaceae were inhibited. Furthermore, changes in concentrations of metabolites, including 4-phenylbutyric acid and phenylacetic acid, were strongly correlated with changes in the abundance of specific gut microbiota. These reciprocal interactions between EGCG and gut microbiota may collectively contribute to the health benefits of EGCG.

KEYWORDS: epigallocatechin-3-gallate, gut microbiota, degradation pathway, UHPLC-Q-Orbitrap-MS, $16 S$ rRNA sequencing

\section{INTRODUCTION}

Green tea, produced from Camellia sinensis, is widely consumed around the world. Numerous epidemiological and pharmacological studies have shown that green tea consumption confers various beneficial effects on human health, including antioxidant, antibacterial, and antiviral activities; body-weight control; and reduction in the risk of cardiovascular disease and some forms of cancer. ${ }^{1}$ These health benefits are generally attributed to the phenolic compounds present in green tea, particularly catechins. ${ }^{2}$ The four most abundant green tea catechins are epicatechin (EC), epicatechin-3-gallate (ECG), epigallocatechin (EGC), and epigallocatechin-3-gallate (EGCG) (Figure 1). ${ }^{3}$ However, it has been established that these compounds are poorly bioavailable in the small intestine. Stalmach et al. reported that approximately $70 \%$ of the ingested green tea catechins were recovered in the large intestine. ${ }^{4}$ EC and EGC reportedly are approximately 31 and $14 \%$ bioavailable, respectively, whereas bioavailability of EGCG, the most abundant catechin in green tea, is very poor (approx. 0.1\%). ${ }^{5}$ The relatively low bioavailability of catechins seems to be in contrast with their beneficial health effects.

After consumption of green tea, the majority of catechins reach the colon, where they are subjected to enzymatic degradation by a large and diverse population of microorganisms. The primary metabolites of catechins after extensive microbial degradation were found to be phenolic acids. $^{6,7}$ These metabolites can be absorbed by the colon and distributed throughout the human body. ${ }^{8}$ Possibly, the health effects derived from green tea consumption are, at least partially, an effect of catechin metabolites rather than the intact<smiles>COc1cc(O)cc(O)c1CC(C)O</smiles><smiles>O=C(OC1Cc2c(O)cc(O)cc2OC1c1ccc(O)c(O)c1)c1cc(O)c(O)c(O)c1</smiles>

Epicatechin gallate $(E C G)$
Epicatechin (EC)<smiles>Oc1cc(O)c2c(c1)OC(c1cc(O)c(O)c(O)c1)C(O)C2</smiles>

Epigallocatechin (EGC)<smiles>O=C(OC1Cc2c(O)cc(O)cc2OC1c1cc(O)c(O)c(O)c1)c1cc(O)c(O)c(O)c1</smiles>

Epigallocatechin gallate (EGCG)
Figure 1. Chemical structures of EC, EGC, ECG, and EGCG.

catechins. For example, it was reported that the catechin C-ring opening product 1-( $3^{\prime}, 4^{\prime}$-dihydroxyphenyl)-3- $\left(2^{\prime \prime}, 4^{\prime \prime}, 6^{\prime \prime}\right.$ trihydroxyphenyl)propan-2-ol had higher antioxidant activity than intact catechin.

Received: June 7, 2020

Revised: July 13, 2020

Accepted: August 18, 2020

Published: August 18, 2020

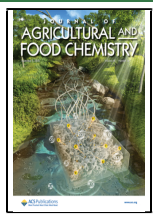


Additionally, it has been widely recognized that gut microbiota play an essential role in the maintenance of intestinal homeostasis and host health. The beneficial bacteria extract nutrients and energy and constitute a physical and immunologic barrier against pathogens. ${ }^{10}$ Hence, gut microbiota composition can affect host health. Therefore, several strategies have been developed to modulate the composition, and thereby the metabolic and immunological activity of the gut microbiota, such as using pro- and prebiotics. ${ }^{11}$ Recent studies show that dietary intervention with phenolic compounds, most notably those from tea, red wine, or cocoa, promotes a more health-beneficial human gut microbiota composition. ${ }^{12-14}$ Several in vitro and in vivo studies have indicated that green tea can promote the growth of beneficial bacteria, such as Bifidobacterium and Lactobacillus, and inhibit pathogenic bacteria, such as Clostridium. ${ }^{15-17}$

Such reciprocal interactions between the catechins and gut microbiota could explain the biological activities of catechins, despite their low bioavailability. However, the majority of studies on this topic focus on either the metabolism of catechins by gut microbiota or the modulatory effects of catechins on gut microbiota composition. The study of both of these aspects within a single experimental setup may provide deeper insights into the reciprocal interactions between tea phenolics and gut microbiota. To the best of our knowledge, such a study has not yet been performed with green tea catechins. Therefore, in the present study, we aimed to investigate the two-way interplay between catechins and gut microbiota. To this end, catechins were subjected to in vitro fermentation by human gut microbiota. Throughout the fermentation, the metabolic fate of catechins as well as the influence of catechins and their metabolites on gut microbiota composition were monitored.

\section{MATERIALS AND METHODS}

Chemicals. Standards of EC, EGC, ECG, EGCG, gallic acid, pyrogallol, benzoic acid, 4-hydroxybenzoic acid, 3-( $3^{\prime}, 4^{\prime}$ dihydroxyphenyl)propionic acid, 2-(4'-hydroxyphenyl)acetic acid, 4phenylbutyric acid, 5-(4'-hydroxyphenyl)valeric acid, 3-(4'hydroxyphenyl)propionic acid, and 5-phenylvaleric acid were purchased from Sigma-Aldrich (St. Louis, MO). Acetonitrile (ACN) was purchased from Biosolve (Valkenswaard, The Netherlands). ULC/MS grade ACN and water, both with $0.1 \%$ (v/v) formic acid, were purchased from Biosolve (Valkenswaard, The Netherlands). Water for purposes other than ultrahigh performance liquid chromatography-mass spectrometry (UHPLC-MS) was prepared using a Milli-Q water purification system (Millipore, Billerica, MA).

In Vitro Fermentation of Catechins with Human Gut Microbiota. The in vitro fecal fermentation of catechins was performed according to the methodology of $\mathrm{Gu}$ et al. ${ }^{18}$ with some modifications. Fecal materials were obtained from four healthy volunteers (three males and one female, 24-38 years), who reported no consumption of tea in the week prior to the donation and declared no antibiotic treatment in the 3 months prior to the donation. Freshly passed feces were immediately transferred to an anaerobic chamber $\left(4 \% \mathrm{H}_{2}, 15 \% \mathrm{CO}_{2}\right.$, and $81 \% \mathrm{~N}_{2}$; Bactron, Cornelius, $\left.\mathrm{OR}\right)$ and mixed with a culture medium at a ratio of $1: 40(\mathrm{w} / \mathrm{v})$. The culture medium was the standard ileal efflux medium (SIEM), which simulates the conditions in the human colon. ${ }^{19}$ The SIEM medium contained $0.4 \%$ (v/v) CHO medium (pectin, xylan, arabinogalactan, amylopectin, and starch; $12 \mathrm{~g} / \mathrm{L}$ each), $40 \%$ (v/v) BCO medium (60 g/L bactopeptone, $60 \mathrm{~g} / \mathrm{L}$ casein, and $1 \mathrm{~g} / \mathrm{L}$ ox-bile), $1.6 \%(\mathrm{v} / \mathrm{v})$ salt mixture solution, $0.8 \%(\mathrm{v} / \mathrm{v}) \mathrm{MgSO}_{4}(50 \mathrm{~g} / \mathrm{l}), 0.4 \%(\mathrm{v} / \mathrm{v})$ cysteine hydrochloride $(40 \mathrm{~g} / \mathrm{L}), 0.08 \%(\mathrm{v} / \mathrm{v})$ vitamin mixture solution, and $10 \%(\mathrm{v} / \mathrm{v})$ MES buffer (1 M, pH 7.0) in water. All medium ingredients were purchased from Tritium Microbiologie (Veldhoven,
The Netherlands). After mixing fresh feces and the culture medium, the resulting fecal slurries from the four volunteers were pooled and homogenized. To obtain a homogeneous human fecal suspension (HFS), the slurry was strained through four layers of cheesecloth. Separate aliquots of $0.9 \mathrm{~mL} \mathrm{HFS}$ were spiked with solutions of each of the four catechins (EC, ECG, EGC, and EGCG) in water at a final catechin concentration of $0.1 \mathrm{mmol} / \mathrm{L}$. The mixtures were then incubated at $37^{\circ} \mathrm{C}$ in the anaerobic chamber for $48 \mathrm{~h}$. After $0,1,2,4$, $6,12,24$, and $48 \mathrm{~h}$ of fermentation, $100 \mu \mathrm{L}$ samples were taken and diluted in $300 \mu \mathrm{L}$ of $\mathrm{ACN}$ to stop fermentation. Following centrifugation $\left(20 \mathrm{~min}, 22000 \mathrm{~g}, 4{ }^{\circ} \mathrm{C}\right.$ ), the supernatant was stored at $-20{ }^{\circ} \mathrm{C}$ until UHPLC-MS analysis.

Separate EGCG fermentation was performed for combined UHPLC-MS and microbiome analysis. For EGCG fermentation, 9 $\mathrm{mL}$ of HFS was mixed with $1 \mathrm{~mL}$ of EGCG in water to a final EGCG concentration of $0.1 \mathrm{mmol} / \mathrm{L}$. As a blank, $1 \mathrm{~mL}$ of water was added to $9 \mathrm{~mL}$ of HFS. All fermentations were performed in triplicate. Mixtures were incubated at $37{ }^{\circ} \mathrm{C}$ in an anaerobic chamber for $72 \mathrm{~h}$. For UHPLC-MS analysis, samples were taken after 0, 2, 4, 6, 9, 12, 24, 48, and $72 \mathrm{~h}$ of fermentation and treated as described previously. For gut microbiota composition analysis, $1 \mathrm{~mL}$ samples were taken at 0,12 , 24,48 , and $72 \mathrm{~h}$ and immediately frozen at $-80{ }^{\circ} \mathrm{C}$ until further bacterial DNA extraction and analysis.

UHPLC-ESI-IT-MS Analysis of the Degradation of Green Tea Catechins. Separation of samples was performed on a Vanquish UHPLC system (Thermo Fisher Scientific, Bremen, Germany) equipped with a binary pump, split loop autosampler, column compartment, and a diode array detector. Samples were separated on an Acquity UHPLC BEH C18 column $(150 \mathrm{~mm} \times 2.1 \mathrm{~mm}, 1.7 \mu \mathrm{m}$; Waters, Milford, MA) with a VanGuard guard column of the same material $(5 \mathrm{~mm} \times 2.1 \mathrm{~mm}, 1.7 \mu \mathrm{m}$; Waters, Milford, MA). The column compartment heater was operated in still air mode at $45{ }^{\circ} \mathrm{C}$, and the postcolumn cooler was set to $40^{\circ} \mathrm{C}$. The injection volume was $1.0 \mu \mathrm{L}$. Mobile phases consisting of $0.1 \%(\mathrm{v} / \mathrm{v})$ formic acid in water (A) and $0.1 \%(\mathrm{v} / \mathrm{v})$ formic acid in ACN (B) were used at a flow rate of $400 \mu \mathrm{L} / \mathrm{min}$. The elution program was set as follows: isocratic at $1 \% \mathrm{~B}$ for $2 \mathrm{~min} ; 2-3 \mathrm{~min}$ linear gradient to $10 \% \mathrm{~B} ; 3-20$ min linear gradient to $60 \% \mathrm{~B} ; 20-23 \mathrm{~min}$ linear gradient to $99 \% \mathrm{~B} ; 23-26 \mathrm{~min}$ isocratic at $99 \%$ B. The mobile phase was adjusted to starting conditions in $1 \mathrm{~min}$, followed by equilibration for $3 \mathrm{~min}$.

A Velos Pro linear ion trap mass spectrometer (Thermo Scientific, San Jose, CA) equipped with a heated electrospray ionization (ESI) probe was coupled to the UHPLC system. Nitrogen was used as the sheath gas (15 arbitrary units) and auxiliary gas (10 arbitrary units). Data were collected in negative ionization mode over the $\mathrm{m} / z$ range of 100-1500. Data-dependent $\mathrm{MS}^{2}$ analysis was performed with a normalized collision energy of $35 \%$. Dynamic exclusion, with a repeat count of 2, repeat duration of $5.0 \mathrm{~s}$, and an exclusion duration of $5.0 \mathrm{~s}$, was used to obtain $\mathrm{MS}^{2}$ spectra of multiple different ions present in full MS at the same time. Most settings were optimized via automatic tuning using LTQ Tune Plus 2.7 (Thermo Scientific, San Jose, CA). The transfer tube temperature was $350{ }^{\circ} \mathrm{C}$, the source heater temperature was $408{ }^{\circ} \mathrm{C}$, and the source voltage was $4.0 \mathrm{kV}$. Data acquisition and reprocessing were performed with Xcalibur software (version 4.1, Thermo Fisher Scientific, Bremen, Germany). External calibration curves $\left(R^{2}>0.99\right)$ of standards of EC, EGC, ECG, and EGCG in the concentration range of 3.125-100 $\mu \mathrm{mol} / \mathrm{L}$ were used to quantify the four catechins.

Ultrahigh Performance Liquid Chromatography Hybrid Quadrupole Orbitrap Mass Spectrometry (UHPLC-Q-Orbitrap-MS) Analysis of the Degradation Pathway of EGCG. For EGCG fermentation, the same UHPLC separation was performed as described previously. A Thermo Q-Exactive Focus hybrid quadrupole Orbitrap mass spectrometer (Thermo Fisher Scientific, Bremen, Germany) equipped with a heated ESI source was coupled to the UHPLC system. Nitrogen was used as the sheath gas (15 arbitrary units) and auxiliary gas (10 arbitrary units). Data were collected in negative and positive ionization modes over the $\mathrm{m} / z$ range of 1001500. The resolution of MS full scan and MS/MS were 70000 and 17500 full width at half-maximum (FWHM), respectively. Instrument 
control and data acquisition were performed with Xcalibur. An external calibration for mass accuracy was carried out before the analysis according to the manufacturer's guidelines. The acquired raw data files of each sample were processed using Compound Discoverer software (version 2.1, Thermo Fisher Scientific, Bremen, Germany). External standards of EC, EGC, ECG, EGCG, gallic acid, pyrogallol, benzoic acid, 4-hydroxybenzoic acid, 3-( $3^{\prime}, 4^{\prime}$-dihydroxyphenyl)propionic acid, 2-(4'-hydroxyphenyl)acetic acid, 4-phenylbutyric acid, 5-(4'-hydroxyphenyl)valeric acid, 3-(4'-hydroxyphenyl)propionic acid, and 5-phenylvaleric acid were used to confirm the identification of metabolites by comparing retention times and accurate masses. The concentrations of these compounds and compounds with similar structures were further calculated based on the respective calibration curves $\left(0.3125\right.$ to $\left.10 \mu \mathrm{mol} / \mathrm{L}, R^{2}>0.99\right)$.

DNA Extraction and High-Throughput Sequencing of Gut Microbiota. Gut microbiota DNA was extracted from each sample using QIAamp Fast DNA Stool Mini Kit (Qiagen, Germany), according to the manufacturer's instructions, and DNA samples were stored at $-20^{\circ} \mathrm{C}$ until used. The quantity and quality of the extracted DNA were checked by $1 \%$ agarose gel electrophoresis. DNA was diluted to $1 \mathrm{ng} / \mu \mathrm{L}$ using sterile water. Bacterial primers 341-F (5'CCT AYG GGR BGC ASC AG-3') and 806-R (5'- GGA CTA CNN GGG TAT CTA AT- $3^{\prime}$ ) with specific barcodes were used to amplify the V3-V4 region of bacterial 16S rRNA genes (466 bp). After amplification, PCR products were checked by $1 \%$ agarose gel electrophoresis. Samples with a bright main strip between 450-550 bp were chosen and purified with QIAquick Gel Extraction Kit (Qiagen, Germany). Subsequently, the sequencing library of the bacterial 16S rRNA genes was generated by utilizing the TruSeq DNA PCR-Free Sample Preparation Kit (Illumina), and the sequencing library was then sequenced on an Illumina HiSeq. 2500 platform.

Statistical Analysis. Raw sequencing reads obtained from the Illumina platform were then merged using FLASH software (Version $1.2 .7)^{20}$ and filtered with QIIME software (Version 1.7). ${ }^{21}$ All quality filtered sequencing reads were then clustered into operational taxonomic units (OTUs) with 97\% sequence similarity, using Uparse software (Version 7.0). ${ }^{22}$ The representative sequence for each bacterial OTU was annotated by comparison against the Silva SSU rRNA database (https://www.arb-silva.de/) with Mothur software (Version 1.30.2). ${ }^{23}$ The relative abundance of each OTU across all samples was calculated and used for further data mining. The overall differences among samples were evaluated by principal component analysis (PCA), using the ade4 package in $\mathrm{R}$ (Version 3.4.0). Subsequently, a linear discriminant analysis effect size (LEfSe) algorithm was performed to identify the representative OTUs characterizing the differences among different groups, using the Huttenhower Lab Galaxy Server (http://huttenhower.sph.harvard. edu/galaxy). The amount of representative OTUs was optimized by adjusting the $\alpha$ value and linear discriminant analysis (LDA) score threshold of the LEfSe analysis. With an $\alpha$ value of 0.05 and an LDA score threshold of 3.1 , a total of 37 representative OTUs were obtained for further analysis. Their relative abundances were further visualized with a heatmap and clustered with hierarchical clustering, using pheatmap package in R. The pairwise Spearman's rho nonparametric correlation analysis between EGCG metabolites and gut microbiota was calculated using Hmisc package in $\mathrm{R}$ and visualized using pheatmap package in $R$.

\section{RESULTS AND DISCUSSION}

General Degradation of the Four Primary Green Tea Catechins. The degradation kinetics of the four catechins by the human gut microbiota in freshly collected fecal samples from four healthy volunteers was first assessed by UHPLCESI-IT-MS. The quantitative changes of the four catechins throughout fermentation and the representative chromatograms (incubation time of $0,2,12,24$, and $48 \mathrm{~h}$ ) of each catechin are shown in Figures 2 and 3, respectively. Degradation of EC, EGC, ECG, and EGCG followed similar

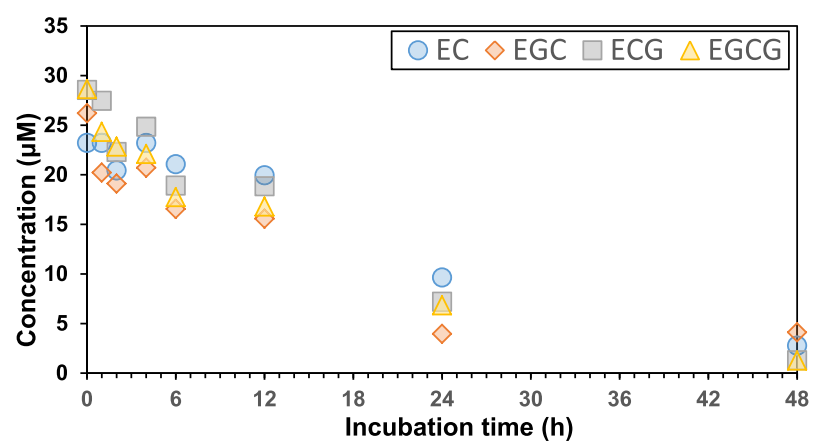

Figure 2. Comparison of the degradation rate of the four catechins by human gut microbiota.

kinetics, starting within $2 \mathrm{~h}$ of fermentation and undergoing the most significant decrease between 12 and $24 \mathrm{~h}$. After $48 \mathrm{~h}$, EC, EGC, ECG, and EGCG were extensively degraded with only $12,15,4$, and $5 \%$ remaining, respectively. Interestingly, all four catechins were promptly degraded by gut microbiota from the initial stage of fermentation. The short survival time of catechins observed in this study was in line with the observations reported by Chen et al. that EC and catechin were both rapidly degraded within $12 \mathrm{~h}$ by rat fecal microbiota. ${ }^{9}$ This indicates that, in vivo, green tea catechins only remain intact for a short period of time upon entering the colon.

Following the decrease of EC $(\mathrm{m} / z 289)$, a peak with $\mathrm{m} / z$ 291 was observed (Figure 3A3-A5). This compound provided three major fragment ions of 247, 167, and 205 (Figure S1, Supporting Information), resulting in a fragmentation spectrum similar to that of EC, albeit at 2 mass units higher, suggesting a catechin-like structure. The $1-2$ bond in the catechin C-ring (Figure 1) is prone to reductive cleavage by several bacteria such as Adlercreutzia equolifaciens, Asaccharobacter celatus, and Slackia equolifaciens, resulting in the formation of a diphenylpropanol. ${ }^{24,25}$ Thus, the peak at $\mathrm{m} / z$ 291 was tentatively identified as $1-\left(3^{\prime}, 4^{\prime}\right.$-dihydroxyphenyl)-3$\left(2^{\prime \prime}, 4^{\prime \prime}, 6^{\prime \prime}\right.$-trihydroxyphenyl)-propan-2-ol. Similar diphenylpropanols were also found during the incubation of EGC $(\mathrm{m} / z$ 305), ECG $(m / z 441)$, and EGCG $(m / z 457)$, namely, 1( $3^{\prime}, 4^{\prime}, 5^{\prime}$-trihydroxyphenyl)-3-(2", $4^{\prime \prime}, 6^{\prime \prime}$-trihydroxyphenyl)propan-2-ol $(\mathrm{m} / z$ 307, Figure 3B3-B5), 1-(3',4'-dihydroxyphenyl)-3-( $2^{\prime \prime}, 4^{\prime \prime}, 6^{\prime \prime}$-trihydroxyphenyl)-propan-2-yl gallate $\left(m / z 443\right.$, Figure 3C5), and 1-( $3^{\prime}, 4^{\prime}, 5^{\prime}$-trihydroxyphenyl)-3$\left(2^{\prime \prime}, 4^{\prime \prime}, 6^{\prime \prime}\right.$-trihydroxyphenyl)-propan-2-yl gallate $(\mathrm{m} / z$ 459, Figure 3D5), respectively. Their $\mathrm{MS}^{2}$ spectra and proposed chemical structures are shown in Figures S2-S4, Supporting Information. For galloylated catechins (ECG and EGCG), degalloylation (i.e., galloyl-ester hydrolysis) was also observed during fermentation, as shown by the formation of EC $(\mathrm{m} / z$ 289 , Figure 2C2-C4) and EGC $(m / z 305$, Figure $2 \mathrm{D} 2-\mathrm{D} 4)$. The combination of degalloylation and $\mathrm{C}$-ring opening was also observed for ECG and EGCG, yielding the EC- and EGCderived diphenylpropanols.

The degalloylated catechins and diphenylpropanols formed as the initial degradation products seemed to be further metabolized (Figure 3). No other notable metabolites could be detected and identified in UHPLC-IT-MS. High-resolution UHPLC-Orbitrap-MS, which is more sensitive, was employed to elucidate the subsequent degradation steps. Due to the similarity of the degradation kinetics and initial degradation 

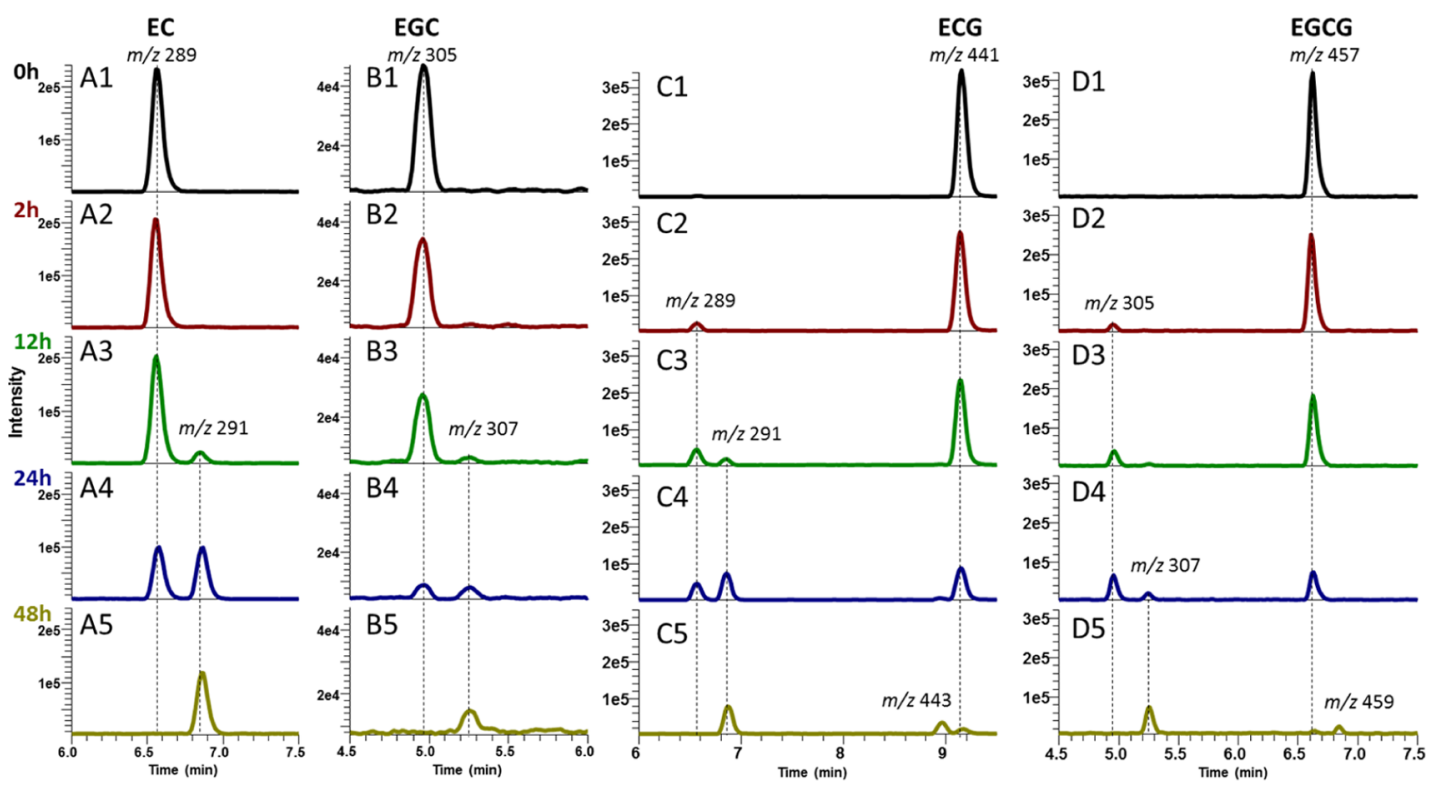

Figure 3. RP-UHPLC-MS base peak chromatograms of eight extracted ions (EC, EGC, ECG, EGCG, and corresponding diphenylpropanols) in negative mode. (A1-A5) EC inoculum samples; (B1-B5) EGC inoculum samples; (C1-C5) ECG inoculum samples; (D1-D5) EGCG inoculum samples; (A1), (B1), (C1), and (D1): 0 h; (A2), (B2), (C2), and (D2): 2 h; (A3), (B3), (C3), and (D3): 12 h; (A4), (B4), (C4), and (D4): $24 \mathrm{~h}$; and (A5), (B5), (C5), and (D5): $48 \mathrm{~h}$.

Table 1. Metabolites of EGCG Fermentation with HFS Annotated by UHPLC-Orbitrap-MS

\begin{tabular}{|c|c|c|c|c|c|c|}
\hline ID & $\mathrm{RT}(\mathrm{min})$ & tentative identification & formula & monoisotopic mass & measured mass & $\Delta$ mass $(\mathrm{ppm})$ \\
\hline M00 & 6.38 & $\mathrm{EGCG}^{a}$ & $\mathrm{C}_{22} \mathrm{H}_{18} \mathrm{O}_{11}$ & 458.08491 & 458.08481 & 0.22 \\
\hline M01 & 5.19 & $\mathrm{EGC}^{a}$ & $\mathrm{C}_{15} \mathrm{H}_{14} \mathrm{O}_{7}$ & 306.07395 & 306.07403 & 0.16 \\
\hline M02 & 2.43 & gallic acid ${ }^{a}$ & $\mathrm{C}_{7} \mathrm{H}_{6} \mathrm{O}_{5}$ & 170.02152 & 170.02147 & 0.29 \\
\hline M03 & 2.80 & pyrogallol $^{a}$ & $\mathrm{C}_{6} \mathrm{H}_{6} \mathrm{O}_{3}$ & 126.03169 & 126.03168 & 0.08 \\
\hline M04 & 6.03 & 1-(3',4'-dihydroxyphenyl)-3-(2", $4^{\prime \prime}, 6^{\prime \prime}$-trihydroxyphenyl)propan-2-ol & $\mathrm{C}_{15} \mathrm{H}_{16} \mathrm{O}_{6}$ & 292.09469 & 292.09460 & 0.31 \\
\hline M05 & 5.12 & 5-(3',4'-dihydroxyphenyl)- $\gamma$-valerolactone & $\mathrm{C}_{11} \mathrm{H}_{12} \mathrm{O}_{4}$ & 208.07356 & 208.07346 & 0.48 \\
\hline M06 & 5.46 & 5 - $\left(3^{\prime}, 4^{\prime}, 5^{\prime}\right.$-trihydroxyphenyl)valeric acid & $\mathrm{C}_{11} \mathrm{H}_{14} \mathrm{O}_{5}$ & 226.08412 & 226.08410 & 0.31 \\
\hline M07 & 13.12 & 4-phenylbutyric acid & $\mathrm{C}_{10} \mathrm{H}_{12} \mathrm{O}_{2}$ & 164.08373 & 164.08372 & 0.06 \\
\hline M08 & 5.85 & 3-(3',4'-dihydroxyphenyl)propionic acid ${ }^{a}$ & $\mathrm{C}_{9} \mathrm{H}_{10} \mathrm{O}_{4}$ & 182.05791 & 182.05788 & 0.16 \\
\hline M09 & 7.07 & 3-(4'-hydroxyphenyl)propionic acid ${ }^{a}$ & $\mathrm{C}_{9} \mathrm{H}_{10} \mathrm{O}_{3}$ & 166.06299 & 166.06291 & 0.48 \\
\hline M10 & 8.28 & 3-phenylpropionic acid & $\mathrm{C}_{9} \mathrm{H}_{10} \mathrm{O}_{2}$ & 150.06808 & 150.06806 & 0.67 \\
\hline M11 & 4.36 & 2-( $\left(3^{\prime}, 4^{\prime}\right.$-dihydroxyphenyl)acetic acid & $\mathrm{C}_{8} \mathrm{H}_{8} \mathrm{O}_{4}$ & 168.04226 & 168.04215 & 0.65 \\
\hline M12 & 5.87 & 2-(4'-hydroxyphenyl)acetic acid ${ }^{a}$ & $\mathrm{C}_{8} \mathrm{H}_{8} \mathrm{O}_{3}$ & 152.04734 & 152.04730 & 0.26 \\
\hline M13 & 9.20 & phenylacetic acid & $\mathrm{C}_{8} \mathrm{H}_{8} \mathrm{O}_{2}$ & 136.05243 & 136.05243 & 0.00 \\
\hline M14 & 5.37 & 4-hydroxybenzoic acid $^{a}$ & $\mathrm{C}_{7} \mathrm{H}_{6} \mathrm{O}_{3}$ & 138.03169 & 138.03167 & 0.14 \\
\hline
\end{tabular}

products among the four catechins, EGCG was selected as the representative catechin for further study.

Complete Degradation Pathway of EGCG by Human Gut Microbiota. The metabolic fate of EGCG during $72 \mathrm{~h}$ of incubation with human gut microbiota was monitored by untargeted UHPLC-Q-Orbitrap-MS analysis. A total of 14 potential metabolites of EGCG were identified, seven of which were confirmed with authentic standards (Table 1). All of these metabolites, except for 2-(4'-hydroxyphenyl)acetic acid and 4-hydroxybenzoic acid, were absent from the blank samples (data not shown). These two exceptions were identified in some blank samples, in which they may have been derived from other food phenolics but at a much lower relative abundance (less than $10 \%$, data not shown) compared to EGCG-treated samples. These 14 metabolites were quantified using either the corresponding authentic standard or an authentic standard with a similar structure. To be specific, EC was used to quantify the concentration of $1-\left(3^{\prime}, 4^{\prime}\right.$ dihydroxyphenyl)-3-(2", $4^{\prime \prime}, 6^{\prime \prime}$-trihydroxyphenyl)propan-2-ol, 5-( $4^{\prime}$-hydroxyphenyl $)$ valeric acid was used to quantify 5- $\left(3^{\prime}, 4^{\prime}\right.$ dihydroxyphenyl)- $\gamma$-valerolactone and $5-\left(3^{\prime}, 4^{\prime}, 5^{\prime}\right.$ trihydroxyphenyl)valeric acid, 3-(4'-hydroxyphenyl)propionic acid was used to quantify 4-phenylpropionic acid, and 2-(4'hydroxyphenyl)acetic acid was used to quantify $4-\left(3^{\prime}, 4^{\prime}\right.$ dihydroxyphenyl)acetic acid and phenylacetic acid. The changes in the content of these 14 metabolites during fermentation of EGCG are shown in Figure 4.

As can be seen in Figure 4 M00-M02, EGCG decreased rapidly during the first $12 \mathrm{~h}$ of fermentation, resulting in formation of EGC and gallic acid, both of which were subject to further metabolism. Pyrogallol was detected as one of the metabolites of gallic acid, which can be formed upon decarboxylation (Figure 4 M03). Pyrogallol concentrations decreased again after $12 \mathrm{~h}$, suggesting further degradation, for 

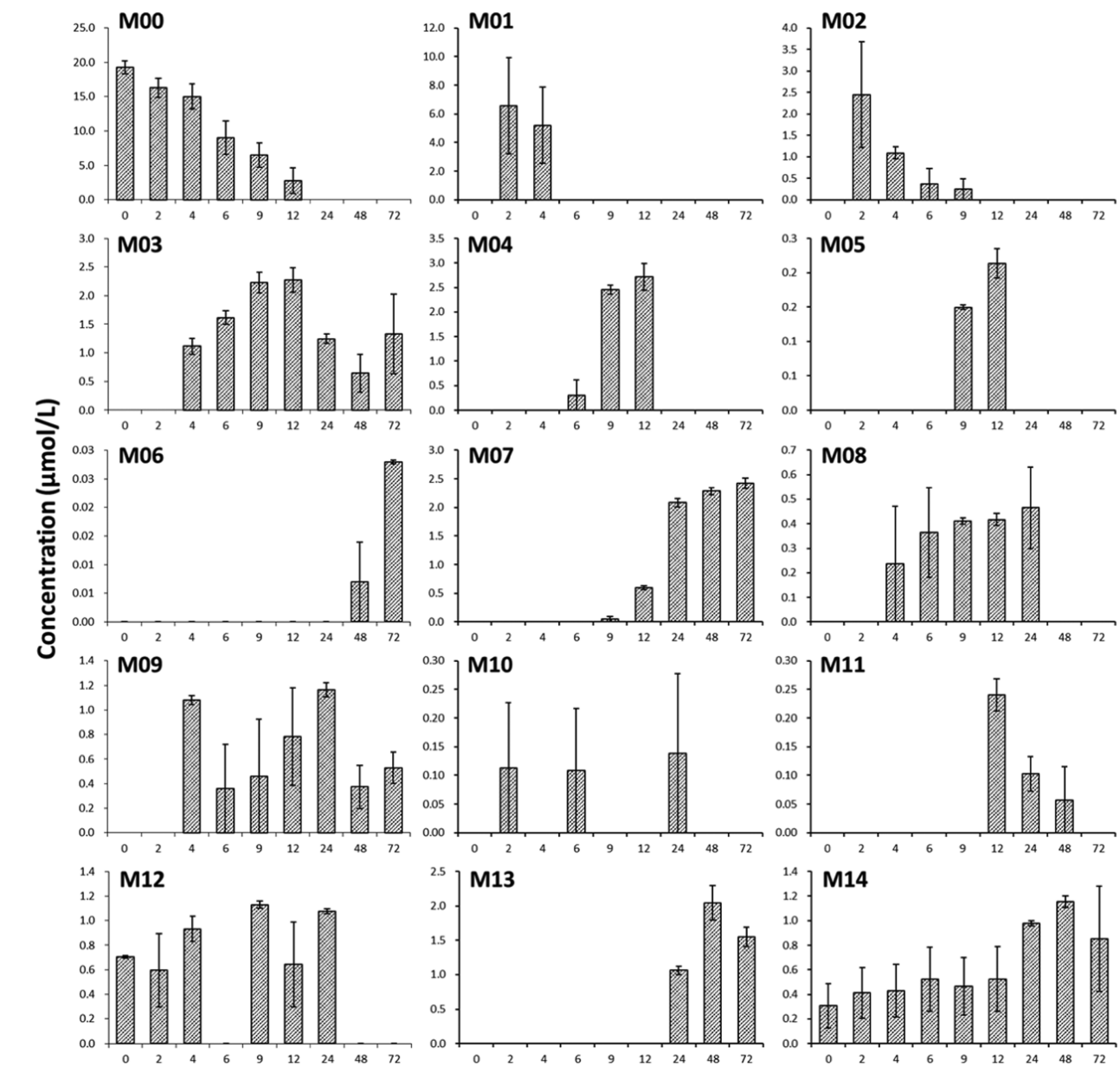

Incubation time (h)

Figure 4. Changes in the concentrations of EGCG and its metabolites during fermentation with human gut microbiota for $72 \mathrm{~h}$ (M00-M14 are listed in Table 1). Error bars indicate standard error $(n=3)$.

example, to butyric acid and acetic acid. ${ }^{26,27}$ An increase in pyrogallol toward the end of the fermentation indicates that it can also be derived via another metabolic route.

Additionally, a peak was detected with a mass corresponding to a C-ring opened and dehydroxylated derivative of EGC, which was tentatively identified as $1-\left(3^{\prime}, 4^{\prime}\right.$-dihydroxyphenyl)3-(2",4",6"-trihydroxyphenyl)propan-2-ol (Figure 4 M04). Further microbial metabolism of this diphenylpropanol led to the formation of a phenylvalerolactone derivative, 5- $\left(3^{\prime}, 4^{\prime}\right.$ dihydroxyphenyl)- $\gamma$-valerolactone (Figure 4 M05), which is formed via A-ring fission, as reported previously by others. ${ }^{6,7}$ The lactone can be opened, resulting in phenylvaleric acids, ${ }^{7}$ explaining the formation of 5-( $3^{\prime}, 4^{\prime}, 5^{\prime}$-trihydroxyphenyl)valeric acid. Phenyl- $\gamma$-valerolactones and phenylvaleric acids were reported as important metabolic intermediates formed from different types of flavan-3-ols. ${ }^{28}$ Subsequent successive alkyl shortening of this hydroxyphenylvaleric acid on the side chain might occur and result in a series of phenylcarboxylic acids with various side chain lengths, such as hydroxyphenylbutyric, hydroxyphenylpropionic, hydroxyphenylacetic, and hydroxybenzoic acids. ${ }^{29}$ In addition, dehydroxylation can also occur at C-3, 4, and 5 of the benzene ring of these hydroxylated phenylcarboxylic acids. Theoretically, a total of 20 possible hydroxylated phenylcarboxylic acids with $1-5$ carbon atoms in the side chain and 0-3 hydroxyl groups on the aromatic ring could be formed via aliphatic chain shortening and dehydroxylation of hydroxyphenylvaleric acid. In the present study, nine such hydroxylated phenylcarboxylic acids were detected, and the changes in the concentrations in each of these compounds are shown in Figure 4 M06-M14. Several studies have shown that phenylcarboxylic acids are rapidly absorbed in the gastrointestinal tract and can exhibit potent antioxidative and anti-inflammatory properties in blood, tissues, or locally in the intestinal lumen. ${ }^{30}$ In addition, Mena et al. highlighted the biological properties of phenyl- $\gamma$ valerolactones and phenylvaleric acids, which include antiinflammatory effects and preventive effects on some chronic diseases. $^{28}$

In summary, EGCG was extensively catabolized by gut microbiota. The initial steps of metabolism include degalloylation, C-ring opening, and A-ring fission, leading to formation of the upstream metabolites, which include diphenylpropanols, phenylvalerolactones, and phenylvaleric acids. Subsequent degradation reactions include aliphatic chain shortening of phenylvaleric acids and the dehydroxylation of the phenyl moiety, leading to the formation of the downstream metabolites, which consist of a series of hydroxylated phenylcarboxylic acids. Enzymes involved in these reactions are most likely microbial esterases, dehydroxylases, and decarboxylases; however, there is limited information available on these enzymes. The complete pathway of EGCG metabolism is summarized in Figure 5. 


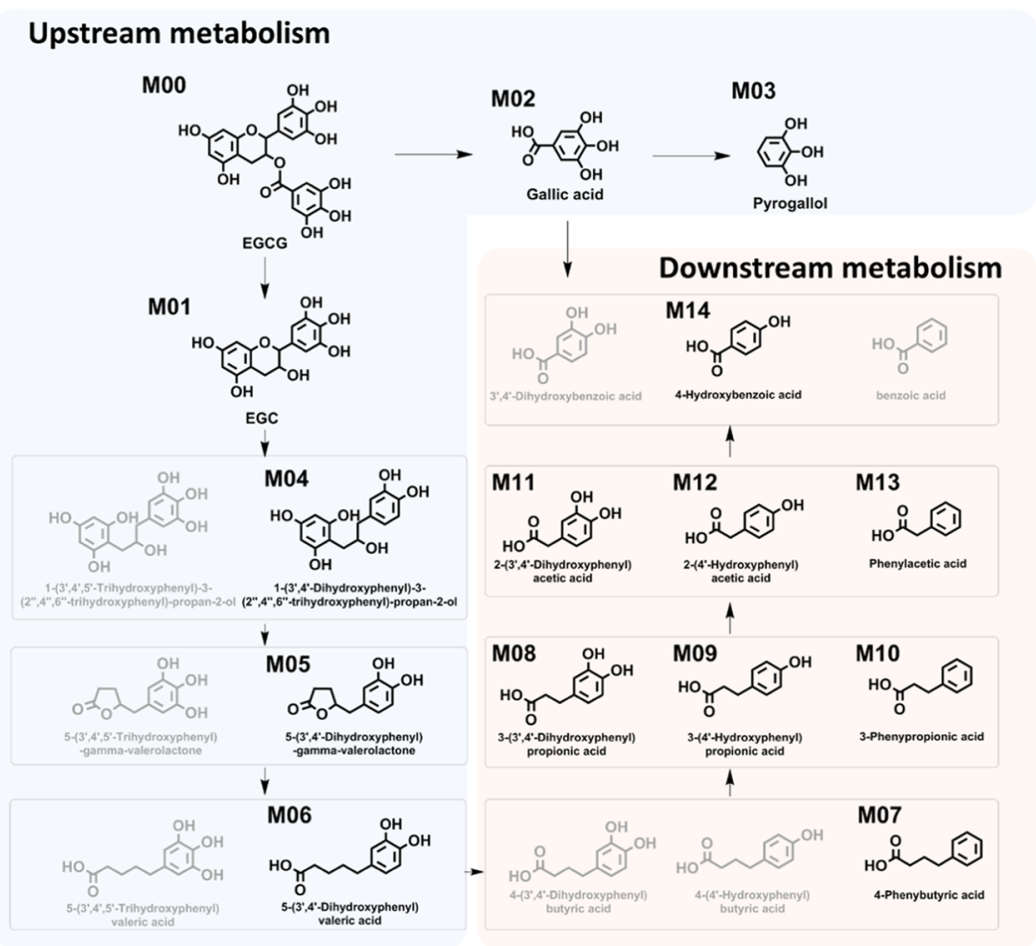

Figure 5. Microbial degradation pathways of EGCG by human gut microbiota. The compounds in black (M00-M14) are the detected metabolites, which are listed in Table 1, and the compounds in gray are theoretical intermediates that were not detected.

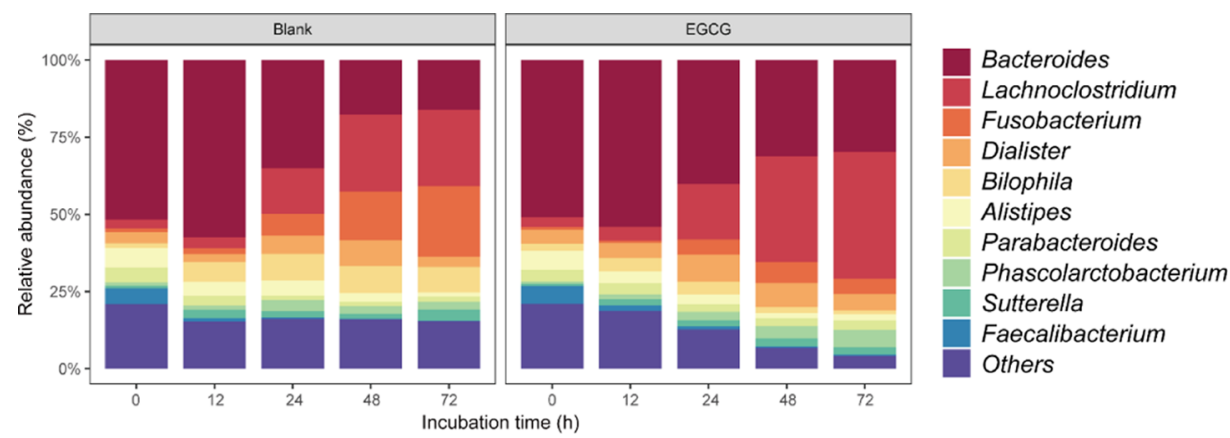

Figure 6. Relative abundances of the most abundant bacterial taxa at the genus level during fermentation without (blank) and with EGCG.

EGCG Changed the Gut Microbiota Composition. The fecal microbiota compositions at fermentation durations of 0 , $12,24,48$, and $72 \mathrm{~h}$ were analyzed using high-throughput bacterial 16S rRNA gene (V3-V4 region) sequencing to evaluate the gut microbiota modulatory effect of EGCG. After merging and filtration of raw sequencing reads, a total of 1719926 reads were generated from the 30 fermentation broth samples (blank and EGCG-treated samples over five time points in triplicate), with an average length of $412 \mathrm{bp}$. After taxonomic annotation, the relative abundance of these OTUs in each sample was used for further analysis. These OTUs were assigned to eight different phyla, whose average relative abundances are depicted in Figure S5, Supporting Information. At the genus level, a total of 211 bacterial genera were identified across all samples. The changes in the average relative abundance of the 10 most abundant genera during incubation are shown in Figure 6. In the EGCG samples, as well as in the blank, a decrease of Bacteroides and an increase of Lachnoclostridium were observed. However, EGCG-treated samples had significantly higher relative abundances of Bacteroides and Lachnoclostridium than the blank $(p<0.05)$.
Thus, the supplementation of EGCG for $72 \mathrm{~h}$ significantly affected the microbiota composition.

To compare the overall differences of gut microbiota among all samples, the relative abundance of all OTUs from each sample was analyzed by PCA, as shown in Figure 7. The first two principal components of PCA accounted for $75.6 \%$ of the total variation, which was sufficient to interpret most of the information of the gut microbiota in each sample. The baseline $(0 \mathrm{~h})$ samples for the blank and EGCG were clustered together, as expected. At $12 \mathrm{~h}$, slight differences between EGCG-treated samples and blank samples were observed, but the samples were still clustered. Thereafter, more pronounced differences between the blank and EGCG samples emerged, leading to the formation of two additional separate clusters in the PCA score plot. These results suggested that the gut microbiota required time to adapt to the presence of EGCG (up to $12 \mathrm{~h}$ ), after which the microbiota composition was modulated by EGCG supplementation.

Identification of the Key EGCG Responding Gut Microbiota. To further identify the gut microbiota most affected by EGCG, a LEfSe analysis was performed. This 


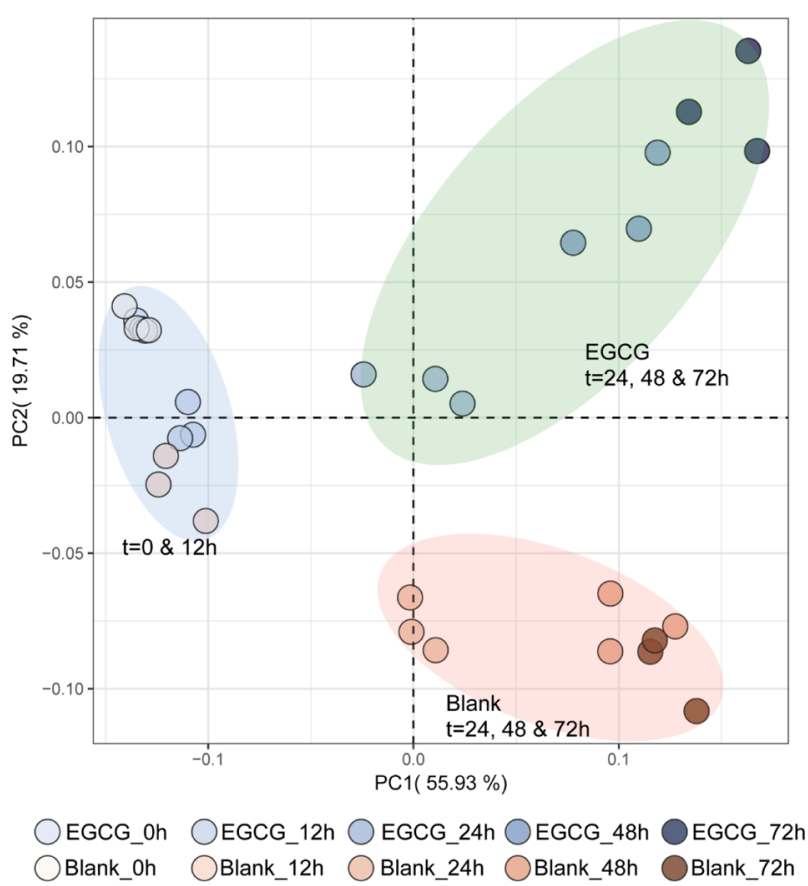

Figure 7. PCA scatter plots based on the relative abundance of bacterial $16 \mathrm{~S}$ gene OTUs of microbial communities in the samples from different fermentation durations $(n=3)$.

algorithm first detects the features with significant differential abundance between two or more data sets using the nonparametric factorial Kruskal-Wallis sum-rank test. Subsequently, it uses linear discriminant analysis (LDA) to estimate the effect size of each differentially abundant feature and returns an LDA score for each feature. The higher LDA score of a certain feature indicates a greater contribution to the discrimination of the data sets. ${ }^{31}$ As the influence of EGCG on gut microbiota was mainly induced after $24 \mathrm{~h}$, LEfSe analysis was applied to the relative abundance of OTUs at fermentation durations of 24,48 , and $72 \mathrm{~h}$, and 37 OTUs were thus identified to differentiate the two groups of samples (Figure $8 \mathrm{~A}$ ). Based on their relative abundance, heatmap plots and hierarchical clustering were then employed, so as to provide a visual and overall comparison for differentiating the two sample groups, as reflected in Figure 8B. With Ward's method, the hierarchical clustering of these OTUs was depicted in a multilayer dendrogram. In the first layer, two groups were clustered, corresponding to the EGCG treatment and the blank. Nine OTUs were significantly more abundant in EGCG-treated samples. These OTUs were considered to be promoted by EGCG supplementation. Twenty-eight OTUs were significantly more abundant in blank samples. These OTUs were considered to be inhibited by EGCG supplementation. The detailed taxonomic information of these 37 OTUs is listed in Table S1, Supporting Information.

Most notably, among these OTUs, five Bacteroides species ( Bacteroides uniformis, Bacteroides vulgatus, Bacteroides stercoris, Bacteroides thetaiotaomicron, and Bacteroides cellulosilyticus) were promoted by EGCG addition as compared to the blank. The genus Bacteroides is one of the dominant genera in human gut microbiota, which constitutes $20-40 \%$ of the human colonic bacteria and imparts substantial metabolic, immunologic, and defensive functions in the gastrointestinal tract. ${ }^{10}$ For example, infant colonic B. uniformis exhibits the
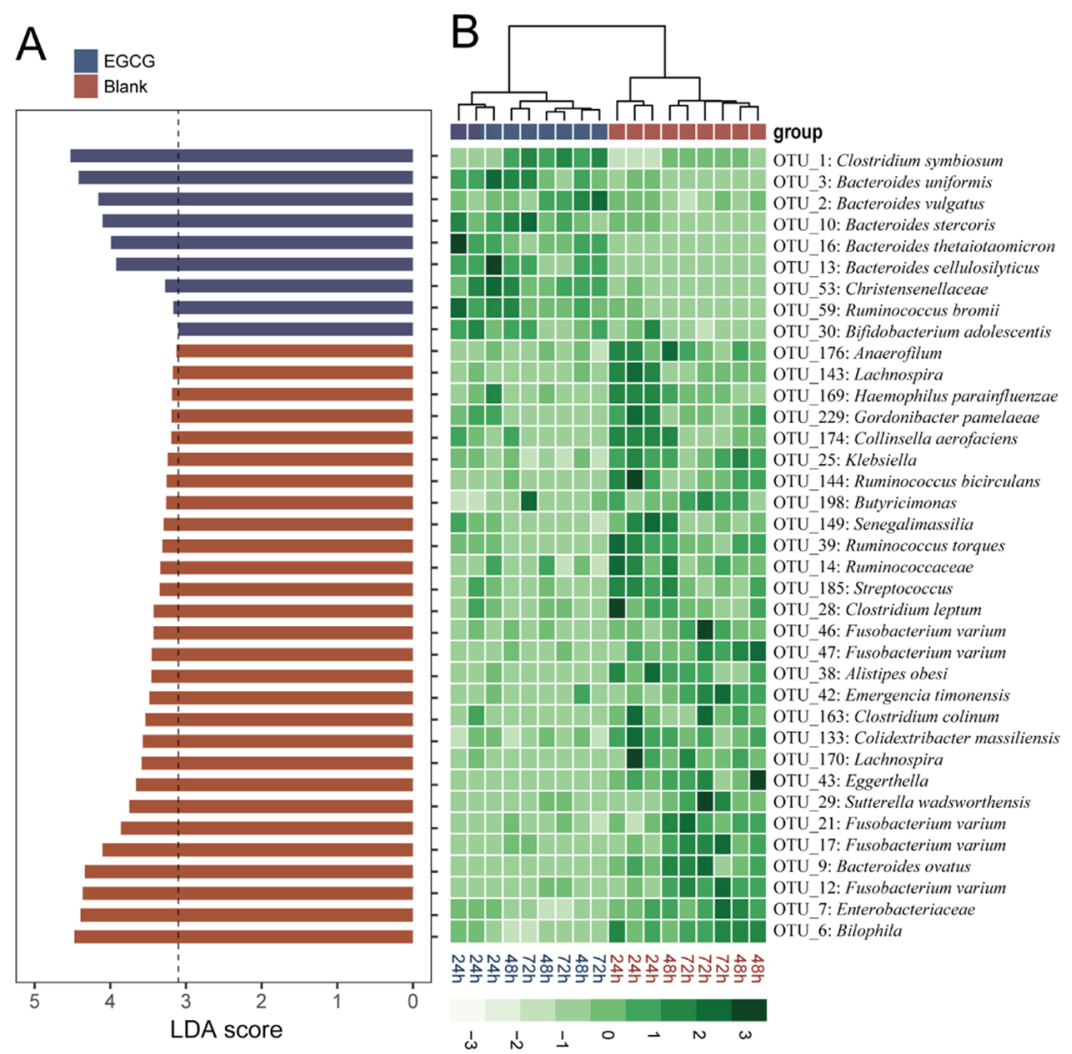

Figure 8. Key OTUs differentiating (LDA score > 3.1) EGCG treatment from the blank. (A) LEfSe comparison between EGCG and blank samples. (B) Heatmap comparison and hierarchical clustering dendrogram based on the relative abundance of the 37 key OTUs. 


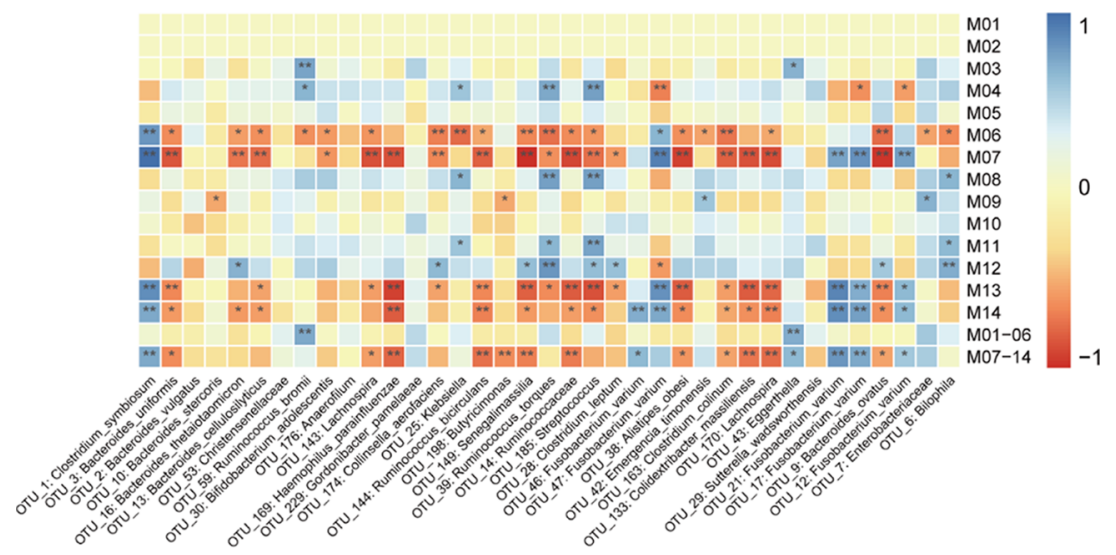

Figure 9. Heatmap of Spearman's correlation between 14 EGCG metabolites and 37 key OTUs affected by EGCG treatment. M01-M14 are listed in Table 1, M01-M06 is the sum of the concentration of the upstream metabolites (M01-M06), and M07-M14 is the sum of the concentration of the downstream metabolites (M07-M14). The colors range from red (negative correlation) to blue (positive correlation). Significant correlations are noted by $*(p<0.05)$ and $* *(p<0.01)$.

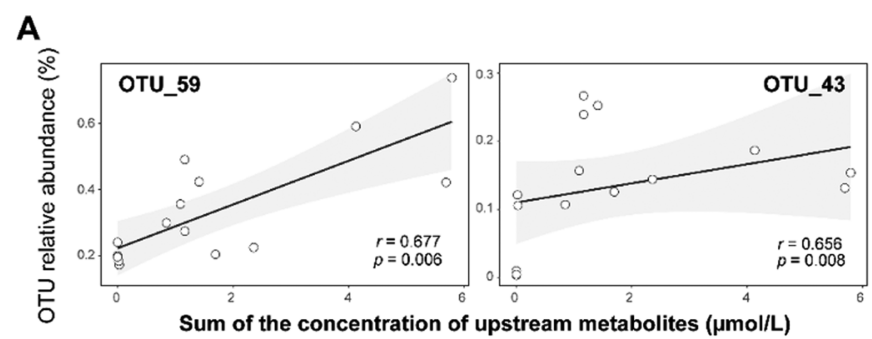

B
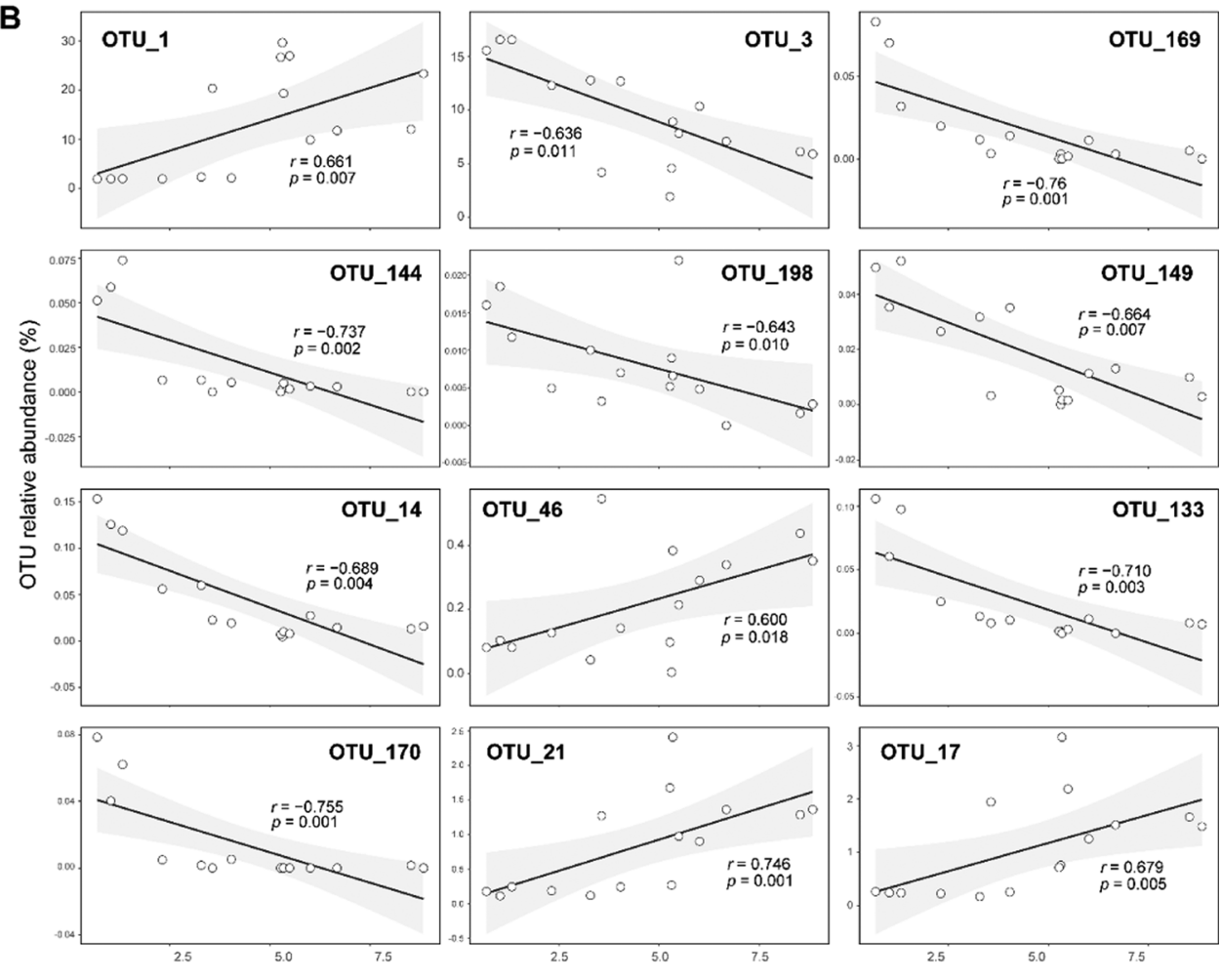

Figure 10. Significant Spearman's correlation $(|r|>0.6, p<0.05)$ between the relative abundance of bacterial OTUs and the sum of the concentration of upstream (A) or downstream (B) metabolites during the incubation of EGCG with human gut microbiota. The fit lines with $95 \%$ confidence bands were generated by linear regression analysis.

ability to boost anti-inflammatory cytokine production and ameliorating metabolic and immune dysfunction. ${ }^{32,33}$ Fecal $B$. vulgatus was found to negatively correlate with coronary artery disease, and it was demonstrated to reduce gut microbial lipopolysaccharide production and thus exert anti-inflammatory $_{\text {properties. }}^{34}$ Due to potent health-promoting benefits, 
several species and strains of genus Bacteroides are now considered as next-generation probiotics. ${ }^{35}$ However, OTU_9, representing Bacteroides ovatus, was found to be inhibited by EGCG treatment, indicating that even within genera EGCG may differentially affect bacterial species.

In addition to the stimulation of these five Bacteroides species, Clostridium symbiosum, Christensenellaceae, Ruminococcus bromii, and Bifidobacterium adolescentis were also increased by EGCG supplementation. Bifidobacteria are well-known probiotics, which are widely used for their beneficial effects on human health. The bacterial family Christensenellaceae is linked to low body mass index and increased longevity. ${ }^{36}$ Thus, Christensenellaceae and B. adolescentis could be considered as potentially beneficial bacteria. C. symbiosum is a butyrateproducing and nontoxin-producing anaerobe, ${ }^{37}$ whereas $R$. bromii is an amylolytic bacteria, which plays an important role in the degradation of dietary resistant starch. ${ }^{38}$ However, their effects on human health are not yet fully understood.

The promotion of Bacteroides and Bifidobacterium by green tea catechins was also reported in other studies. ${ }^{16,39,40}$ It was also reported that some other beneficial gut microbiota, such as Akkermansia and Lactobacillus, were increased by green tea; ${ }^{16,41}$ however, we did not observe this in our study. The promotion of five Bacteroides species and several other healthpromoting species by the addition of EGCG might indicate that EGCG supplementation in food contributes to overall improved gut health.

In contrast, five OTUs representing Forsterygion varium (OTU_12, 17, 21, 46, and 47) were repressed by EGCG treatment. This microorganism can invade colonic epithelial cells and initiate a proinflammatory response, and thus is considered to be a gastrointestinal pathogen. ${ }^{42}$ Similarly, the inhibitory effect of EGCG on several other harmful bacteria was observed, such as Bilophila (OTU_6), a hydrogen-sulfideproducing colonic virulent microorganism. ${ }^{43}$ Moreover, Enterobacteriaceae (OTU_7) were reduced upon EGCG treatment; this large bacterial family includes pathogenic organisms, such as Salmonella, Escherichia coli, Yersinia pestis, Klebsiella, Shigella, and Eggerthella (OTU_43), which are implicated as a cause of ulcerative colitis, liver and anal abscesses, and systemic bacteraemia. $^{44}$

Correlation between EGCG Metabolites and Gut Microbiota. To preliminarily assess the reciprocal effects between EGCG metabolism and modulation of the gut microbiota composition, correlations between the EGCG metabolites and the 37 key OTUs were assessed by Spearman correlation analysis (Figure 9). To this end, the correlations of the 14 detected metabolites were assessed individually and divided into two groups: six upstream metabolites (M01M06) and eight downstream metabolites (M07-M14). Positive correlations between metabolite concentration and microbial OTU abundance suggest that the bacteria may be involved in the production of the metabolites or that their growth is stimulated by the metabolites. Negative correlations imply that the bacteria may be inhibited by the metabolites or that the metabolites are consumed or further metabolized by the bacteria. The results indicated that the upstream metabolites showed high correlation with two bacterial taxa ( R. bromii and Eggerthella, $r>0.60, p<0.01$, Figure 10A), whereas 12 bacterial taxa were positively or negatively correlated with the downstream metabolites $(|r|>0.60, p<$ 0.05 , Figure 10B). Notably, three of the downstream metabolites (M07, M13, and M14) presented significantly strong correlations with various gut microbiota $(|r|>0.80, p<$ $0.001)$. Specifically, 4-phenylbutyric acid (M07) positively or negatively correlated with 11 bacterial taxa (Figure S6, Supporting Information). Phenylacetic acid (M13) showed a significant positive or negative correlation with five bacterial taxa (Figure S7, Supporting Information). 4-Hydroxybenzoic acid (M14) showed a significant negative correlation with Haemophilus parainfluenzae (Figure S8, Supporting Information).

The initial steps of degradation of EGCG mainly include degalloylation, C-ring opening, and A-ring fission. Cleavage of the ester linkage between gallic acid and flavan-3-ol by microbial esterases, which are widely distributed in microorganisms, has been well documented. ${ }^{45}$ It has been reported that reductive cleavage of the C-ring can be performed by several bacteria, such as Lactobacillus plantarum IFPL935, ${ }^{46}$ Eggerthella lenta, and Flavonifractor plautii. ${ }^{47}$ In this study, the genus Eggerthella was also found to have a positive correlation with the upstream metabolites (Figure 10A). The bacterial species responsible for the reactions involved in the formation of downstream metabolites, i.e., A-ring fission and the subsequent chain shortening and dehydroxylation, have not yet been identified. In this study, the formation of downstream metabolites was found to be highly correlated with multiple gut microbiota (Figure 10B). These findings imply that formation of downstream metabolites might require a diverse set of bacteria that cooperates simultaneously or sequentially to complete the degradation of EGCG. The experimental approach presented in this study, featuring the comprehensive simultaneous determination of metabolites and gut microbiota composition, provides valuable leads for follow-up studies.

Several limitations need to be considered regarding this experimental approach. First, EGCG was applied directly for fermentation by fecal microbiota. In practice, a small percentage of ingested catechins is absorbed by enterocytes and subject to phase II metabolism, resulting in glucuronidation, methylation, and sulfonation. ${ }^{48}$ These conjugates can be returned to the intestinal lumen via efflux. ${ }^{48}$ However, considering the low abundance of these conjugates in the colon, this study focusses on intact catechins as the starting point of gut microbial fermentation. Second, a short-term batch fermentation model was utilized in this study to facilitate sampling at different time points and to gain better insights into the interactions taking place between catechins and gut microbiota. Changes in microbiota composition were observed over time, even in the absence of EGCG. For follow-up studies, we suggest evaluation of these results in more intricate in vitro models, such as simulation of the human intestinal microbial ecosystem (SHIME) ${ }^{49}$ or TNO intestinal models (TIMs). ${ }^{50}$ Third, the role of human intestinal immunity has not been taken into account in this study. Both catechins and their microbial metabolites are known to be able to act as immunomodulators. ${ }^{51,52}$ Modification of the colonic immune status will further influence the gut microbiota composition.

In conclusion, we investigated the reciprocal interactions between EGCG and human gut microbiota. The results indicated that EGCG, as well as other green tea catechins, was prone to metabolism by human gut microbiota. The main microbial metabolites formed via consecutive ester hydrolysis, C-ring opening, A-ring fission, dehydroxylation, and aliphatic chain shortening are phenylcarboxylic acids. Due to the short survival time of intact catechins during fermentation, combined with their poor absorption, we speculate that their metabolites 
may play an important role in the health benefits associated with tea consumption. Simultaneously, the composition of the gut microbiota was altered by EGCG toward a healthier profile. We further explored the possible correlations between EGCG metabolites and gut microbiota and found that a wide range of gut microbiota may be involved in the downstream metabolism of EGCG. The experimental approach and findings described in this study provide novel insights into the reciprocal interactions between EGCG and gut microbiota, which are valuable leads for follow-up studies on the health benefits of EGCG and other green tea phenolics.

\section{ASSOCIATED CONTENT}

\section{SI Supporting Information}

The Supporting Information is available free of charge at https://pubs.acs.org/doi/10.1021/acs.jafc.0c03587.

$\mathrm{MS}^{2}$ spectrum and proposed chemical structure of ECderived diphenylpropanol (Figure S1); EGC-derived diphenylpropanol (Figure S2); ECG-derived diphenylpropanol (Figure S3); EGCG-derived diphenylpropanol (Figure S4); relative abundances of the most abundant bacterial taxa at the phylum level during fermentation without (blank) and with EGCG (Figure S5); significant Spearman's correlation between the relative abundance of bacterial OTUs and the concentration of 4-phenylbutyric acid (Figure S6); phenylacetic acid (Figure S7); 4-hydroxybenzoic acid (Figure S8) during the incubation of EGCG with human gut microbiota; taxonomic information of the $37 \mathrm{key}$ OTUs that differentiated the EGCG treatment from the blank (Table S1) (PDF)

\section{AUTHOR INFORMATION}

\section{Corresponding Author}

Jean-Paul Vincken - Laboratory of Food Chemistry,

Wageningen University, 6700 AA Wageningen, The

Netherlands; 10 orcid.org/0000-0001-8540-4327;

Phone: +31-317482234; Email: jean-paul.vincken@wur.nl

\section{Authors}

Zhibin Liu - Laboratory of Food Chemistry, Wageningen University, 6700 AA Wageningen, The Netherlands; Institute of Food Science \& Technology, Fuzhou University, Fuzhou 350108, P. R. China; (i) orcid.org/0000-0002-8674-7281

Wouter J. C. de Bruijn - Laboratory of Food Chemistry, Wageningen University, 6700 AA Wageningen, The Netherlands; (1) orcid.org/0000-0003-0564-9848

Marieke E. Bruins - Food \& Biobased Research, Wageningen University \& Research, 6700 AA Wageningen, The Netherlands

Complete contact information is available at:

https://pubs.acs.org/10.1021/acs.jafc.0c03587

\section{Funding}

Z.L. acknowledges the financial support from the China Scholarship Council (CSC).

\section{Notes}

The authors declare no competing financial interest.

\section{ACKNOWLEDGMENTS}

The authors thank Mark Sanders of the Laboratory of Food Chemistry, Wageningen University, for his excellent technical assistance.

\section{ABBREVIATIONS USED}

CAN, acetonitrile; EC, epicatechin; ECG, epicatechin-3gallate; EGC, epigallocatechin; EGCG, epigallocatechin-3gallate; ESI, electrospray ionization; FWHM, full width at half-maximum; HFS, human fecal suspension; LDA, linear discriminant analysis; LEfSe, linear discriminant analysis effect size; OTU, operational taxonomic unit; PCA, principal component analysis; SIEM, standard ileal efflux medium; UHPLC-Q-Orbitrap-MS, ultrahigh performance liquid chromatography hybrid quadrupole Orbitrap mass spectrometry

\section{REFERENCES}

(1) Cabrera, C.; Artacho, R.; Giménez, R. Beneficial effects of green tea-a review. J. Am. Coll. Nutr. 2006, 25, 79-99.

(2) Zaveri, N. T. Green tea and its polyphenolic catechins: medicinal uses in cancer and noncancer applications. Life Sci. 2006, 78, 20732080.

(3) Liu, Z.; Bruins, M. E.; de Bruijn, W. J.; Vincken, J.-P. A comparison of the phenolic composition of old and young tea leaves reveals a decrease in flavanols and phenolic acids and an increase in flavonols upon tea leaf maturation. J. Food Compos. Anal. 2020, 86, No. 103385.

(4) Stalmach, A.; Mullen, W.; Steiling, H.; Williamson, G.; Lean, M. E. J.; Crozier, A. Absorption, metabolism, and excretion of green tea flavan-3-ols in humans with an ileostomy. Mol. Nutr. Food Res. 2010, 54, 323-334.

(5) Chen, L.; Lee, M.-J.; Li, H.; Yang, C. S. Absorption, distribution, and elimination of tea polyphenols in rats. Drug Metab. Dispos. 1997, $25,1045-1050$.

(6) Takagaki, A.; Nanjo, F. Catabolism of (+)-catechin and (-)-epicatechin by rat intestinal microbiota. J. Agric. Food. Chem. 2013, 61, 4927-4935.

(7) Sánchez-Patán, F.; Cueva, C.; Monagas, M.; Walton, G. E.; Gibson, G. R.; Martín-Álvarez, P. J.; Moreno-Arribas, M. V.; Bartolomé, B. Gut microbial catabolism of grape seed flavan-3-ols by human faecal microbiota. Targetted analysis of precursor compounds, intermediate metabolites and end-products. Food Chem. 2012, 131, 337-347.

(8) Chen, H.; Sang, S. Biotransformation of tea polyphenols by gut microbiota. J. Funct. Foods 2014, 7, 26-42.

(9) Chen, W.; Zhu, X.; Lu, Q.; Zhang, L.; Wang, X.; Liu, R. C-ring cleavage metabolites of catechin and epicatechin enhanced antioxidant activities through intestinal microbiota. Food Res. Int. 2020, 135, No. 109271.

(10) Jandhyala, S. M.; Talukdar, R.; Subramanyam, C.; Vuyyuru, H.; Sasikala, M.; Reddy, D. N. Role of the normal gut microbiota. World J. Gastroenterol. 2015, 21, 8787.

(11) Pandey, K. R.; Naik, S. R.; Vakil, B. V. Probiotics, prebiotics and synbiotics-a review. J. Food Sci. Technol. 2015, 52, 7577-7587.

(12) Marchesi, J. R.; Adams, D. H.; Fava, F.; Hermes, G. D.; Hirschfield, G. M.; Hold, G.; Quraishi, M. N.; Kinross, J.; Smidt, H.; Tuohy, K. M.; et al. The gut microbiota and host health: a new clinical frontier. Gut 2016, 65, 330-339.

(13) Nash, V.; Ranadheera, C. S.; Georgousopoulou, E. N.; Mellor, D.; Panagiotakos, D. B.; McKune, A.; Kellett, J.; Naumovski, N. The effects of grape and red wine polyphenols on gut microbiota-A systematic review. Food Res. Int. 2018, 113, 277-287.

(14) Liu, Z.; Bruins, M. E.; Ni, L.; Vincken, J.-P. Green and black tea phenolics: bioavailability, transformation by colonic microbiota, and modulation of colonic microbiota. J. Agric. Food. Chem. 2018, 66, 8469-8477.

(15) Zhang, X.; Zhu, X.; Sun, Y.; Hu, B.; Sun, Y.; Jabbar, S.; Zeng, X. Fermentation in vitro of EGCG, GCG and EGCG3" Me isolated from oolong tea by human intestinal microbiota. Food Res. Int. 2013, 54, 1589-1595.

(16) Liu, Z.; Chen, Z.; Guo, H.; He, D.; Zhao, H.; Wang, Z.; Zhang, W.; Liao, L.; Zhang, C.; Ni, L. The modulatory effect of infusions of 
green tea, oolong tea, and black tea on gut microbiota in high-fatinduced obese mice. Food Funct. 2016, 7, 4869-4879.

(17) Tzounis, X.; Vulevic, J.; Kuhnle, G. G. C.; George, T.; Leonczak, J.; Gibson, G. R.; Kwik-Uribe, C.; Spencer, J. P. E. Flavanol monomer-induced changes to the human faecal microflora. Br. J. Nutr. 2008, 99, 782-792.

(18) Gu, F.; Borewicz, K.; Richter, B.; van der Zaal, P. H.; Smidt, H.; Buwalda, P. L.; Schols, H. A. In vitro fermentation behavior of isomalto/malto-polysaccharides using human fecal inoculum indicates prebiotic potential. Mol. Nutr. Food Res. 2018, 62, No. 1800232.

(19) Ramasamy, U. S.; Venema, K.; Schols, H. A.; Gruppen, H. Effect of soluble and insoluble fibers within the in vitro fermentation of chicory root pulp by human gut bacteria. J. Agric. Food. Chem. 2014, 62, 6794-6802.

(20) Magoč, T.; Salzberg, S. L. FLASH: fast length adjustment of short reads to improve genome assemblies. Bioinformatics 2011, 27, 2957-2963.

(21) Caporaso, J. G.; Kuczynski, J.; Stombaugh, J.; Bittinger, K.; Bushman, F. D.; Costello, E. K.; Fierer, N.; Pena, A. G.; Goodrich, J. K.; Gordon, J. I. QIIME allows analysis of high-throughput community sequencing data. Nat. Methods 2010, 7, 335 .

(22) Edgar, R. C. UPARSE: highly accurate OTU sequences from microbial amplicon reads. Nat. Methods 2013, 10, 996.

(23) Quast, C.; Pruesse, E.; Yilmaz, P.; Gerken, J.; Schweer, T.; Yarza, P.; Peplies, J.; Glöckner, F. O. The SILVA ribosomal RNA gene database project: improved data processing and web-based tools. Nucleic Acids Res. 2012, 41, D590-D596.

(24) Takagaki, A.; Nanjo, F. Biotransformation of (-)-epigallocatechin and $(-)$-gallocatechin by intestinal bacteria involved in isoflavone metabolism. Biol. Pharm. Bull. 2015, 38, 325-330.

(25) Takagaki, A.; Kato, Y.; Nanjo, F. Isolation and characterization of rat intestinal bacteria involved in biotransformation of (-)-epigallocatechin. Arch. Microbiol. 2014, 196, 681-695.

(26) Krumholz, L. R.; Crawford, R. L.; Hemling, M. E.; Bryant, M. P. Metabolism of gallate and phloroglucinol in Eubacterium oxidoreducens via 3-hydroxy-5-oxohexanoate. J. Bacteriol. 1987, 169, $1886-1890$

(27) Gross, G.; Jacobs, D. M.; Peters, S.; Possemiers, S.; van Duynhoven, J.; Vaughan, E. E.; Van de Wiele, T. In vitro bioconversion of polyphenols from black tea and red wine/grape juice by human intestinal microbiota displays strong interindividual variability. J. Agric. Food. Chem. 2010, 58, 10236-10246.

(28) Mena, P.; Bresciani, L.; Brindani, N.; Ludwig, I. A.; PereiraCaro, G.; Angelino, D.; Llorach, R.; Calani, L.; Brighenti, F.; Clifford, M. N.; et al. Phenyl $\gamma$-valerolactones and phenylvaleric acids, the main colonic metabolites of flavan-3-ols: Synthesis, analysis, bioavailability, and bioactivity. Nat. Prod. Rep. 2019, 36, 714-752.

(29) Selma, M. V.; Espin, J. C.; Tomas-Barberan, F. A. Interaction between phenolics and gut microbiota: role in human health. J. Agric. Food. Chem. 2009, 57, 6485-6501.

(30) Lafay, S.; Gil-Izquierdo, A. Bioavailability of phenolic acids. Phytochem. Rev. 2008, 7, No. 301.

(31) Segata, N.; Izard, J.; Waldron, L.; Gevers, D.; Miropolsky, L.; Garrett, W. S.; Huttenhower, C.; et al. Metagenomic biomarker discovery and explanation. Genome Biol. 2011, 12, No. R60.

(32) Sánchez, E.; De Palma, G.; Capilla, A.; Nova, E.; Pozo, T.; Castillejo, G.; Varea, V.; Marcos, A.; Garrote, J. A.; Polanco, I.; et al. Influence of environmental and genetic factors linked to celiac disease risk on infant gut colonization by Bacteroides species. Appl. Environ. Microbiol. 2011, 77, 5316-5323.

(33) Cano, P. G.; Santacruz, A.; Moya, Á.; Sanz, Y. Bacteroides uniformis CECT 7771 ameliorates metabolic and immunological dysfunction in mice with high-fat-diet induced obesity. PLoS One 2012, 7, No. e41079.

(34) Yoshida, N.; Emoto, T.; Yamashita, T.; Watanabe, H.; Hayashi, T.; Tabata, T.; Hoshi, N.; Hatano, N.; Ozawa, G.; Sasaki, N.; et al. Bacteroides vulgatus and Bacteroides dorei reduce gut microbial lipopolysaccharide production and inhibit atherosclerosis. Circulation 2018, 138, 2486-2498.
(35) Tan, H.; Zhai, Q; Chen, W. Investigations of Bacteroides spp. towards next-generation probiotics. Food Res. Int. 2018, 116, 637644

(36) Kong, F.; Hua, Y.; Zeng, B.; Ning, R.; Li, Y.; Zhao, J. Gut microbiota signatures of longevity. Curr. Biol. 2016, 26, R832-R833.

(37) Kaneuchi, C.; Watanabe, K.; Terada, A.; Benno, Y.; Mitsuoka, T. Taxonomic study of Bacteroides clostridiiformis subsp. clostridiiformis (Burri and Ankersmit) Holdeman and Moore and of related organisms: proposal of Clostridium clostridiiformis (Burri and Ankersmit) comb. nov. and Clostridium symbiosum (Stevens) comb. nov. Int. J. Syst. Evol. Microbiol. 1976, 26, 195-204.

(38) Mukhopadhya, I.; Morais, S.; Laverde-Gomez, J.; Sheridan, P. O.; Walker, A. W.; Kelly, W.; Klieve, A. V.; Ouwerkerk, D.; Duncan, S. H.; Louis, P.; et al. Sporulation capability and amylosome conservation among diverse human colonic and rumen isolates of the keystone starch-degrader Ruminococcus bromii. Environ. Microbiol. 2018, 20, 324-336.

(39) Wang, J.; Tang, L.; Zhou, H.; Zhou, J.; Glenn, T. C.; Shen, C.L.; Wang, J.-S. Long-term treatment with green tea polyphenols modifies the gut microbiome of female sprague-dawley rats. J. Nutr. Biochem. 2018, 56, 55-64.

(40) Unno, T.; SAkUMA, M.; Mitsuhashi, S. Effect of dietary supplementation of (-)-epigallocatechin gallate on gut microbiota and biomarkers of colonic fermentation in rats. J. Nutr. Sci. Vitaminol. 2014, 60, 213-219.

(41) Axling, U.; Olsson, C.; Xu, J.; Fernandez, C.; Larsson, S.; Ström, K.; Ahrné, S.; Holm, C.; Molin, G.; Berger, K. Green tea powder and Lactobacillus plantarum affect gut microbiota, lipid metabolism and inflammation in high-fat fed C57BL/6J mice. Nutr. Metab. 2012, 9, No. 105.

(42) Allen-Vercoe, E. Fusobacterium varium in ulcerative colitis: is it population-based? Dig. Dis. Sci. 2015, 60, 7-8.

(43) Baron, E. J. Bilophila wadsworthia: a unique Gram-negative anaerobic rod. Anaerobe 1997, 3, 83-86.

(44) Lau, S. K. P.; Woo, P. C. Y.; Woo, G. K. S.; Fung, A. M. Y.; Wong, M. K. M.; Chan, K.-m.; Tam, D. M. W.; Yuen, K.-y. Eggerthella hongkongensis sp. nov. and Eggerthella sinensis sp. nov., two novel Eggerthella species, account for half of the cases of Eggerthella bacteremia. Diagn. Microbiol. Infect. Dis. 2004, 49, 255-263.

(45) Braune, A.; Blaut, M. Bacterial species involved in the conversion of dietary flavonoids in the human gut. Gut Microbes 2016, 7, 216-234.

(46) Sánchez-Patán, F.; Tabasco, R.; Monagas, M.; Requena, T.; Pela'ez, C.; Moreno-Arribas, M. V.; Bartolome', Ba. Capability of Lactobacillus plantarum IFPL935 to catabolize flavan-3-ol compounds and complex phenolic extracts. J. Agric. Food. Chem. 2012, 60, 71427151.

(47) Kutschera, M.; Engst, W.; Blaut, M.; Braune, A. Isolation of catechin-converting human intestinal bacteria. J. Appl. Microbiol. 2011, 111, 165-175.

(48) Actis-Goretta, L.; Lévèques, A.; Rein, M.; Teml, A.; Schäfer, C.; Hofmann, U.; Li, H.; Schwab, M.; Eichelbaum, M.; Williamson, G. Intestinal absorption, metabolism, and excretion of (-)-epicatechin in healthy humans assessed by using an intestinal perfusion technique. Am. J. Clin. Nutr. 2013, 98, 924-933.

(49) Alander, M.; De Smet, I.; Nollet, L.; Verstraete, W.; von Wright, A.; Mattila-Sandholm, T. The effect of probiotic strains on the microbiota of the Simulator of the Human Intestinal Microbial Ecosystem (SHIME). Int. J. Food Microbiol. 1999, 46, 71-79.

(50) Venema, K.; Van den Abbeele, P. Experimental models of the gut microbiome. Best Pract. Res., Clin. Gastroenterol. 2013, 27, 115126.

(51) Krishnamoorthy, R.; Adisa, A. R.; Periasamy, V. S.; Athinarayanan, J.; Pandurangan, S.-B.; Alshatwi, A. A. Colonic bacteria-transformed catechin metabolite response to cytokine production by human peripheral blood mononuclear cells. Biomolecules 2019, 9, No. 830. 
(52) Fan, F.-Y.; Sang, L.-X.; Jiang, M. Catechins and their therapeutic benefits to inflammatory bowel disease. Molecules 2017, 22, No. 484. 